\title{
HAADF STEM and PL Characterization of Monolayer-Thick GaN/(Al,Ga)N Quantum Wells for Deep UV Optoelectronics Applications
}

\author{
A.A. Toropov ${ }^{1}$, E.A. Evropeytsev ${ }^{1}$, V.N. Jmerik ${ }^{1}$, D.V. Nechaev ${ }^{1}$, S.V. Ivanov ${ }^{1}$, and S. Rouvimov ${ }^{2}$ \\ 1. Ioffe Institute, Polytekhnicheskaya 26, St. Petersburg, Russia \\ 2. University of Notre Dame, Notre Dame, Indiana, USA
}

A few monolayer-thick (ML) GaN quantum well (QW) structures are promising for high-power optoelectronic applications in mid-UV wavelength ranges [1-2]. High Resolution Transmission Electron Microscopy (HRTEM) and especially High Angle Annual Dark Field (HAADF) Transmission Electron Microscopy (STEM) appear to be a key metrology to establish a relationship between atomic structure and optical properties of nanostructures. TEM/STEM also provides an effective pathway for optimization of crystal growth of ML thick nanostructures [1]. Here we report TEM analysis of 1-5 ML thick GaN QWs in conjunction with optical research of peculiarities of their electronic band structure.

The samples were grown on $c$-sapphire by plasma-assisted molecular beam epitaxy (PA MBE). An accurate control of QW thickness at a sub-monolayer (ML) resolution has been achieved by growth of both barrier AlN and well GaN layers at the metal-rich conditions and low substrate temperatures using different pulsed growth techniques such as metal-modulated and migration-enhanced epitaxy. Time-resolved (TR) photoluminescence (PL) spectroscopy has been employed to study optical properties of these samples. The HAADF STEM analysis has been carried out on FEI Titan 80-300 TEM with special resolution of $1.3 \mathrm{~A}$.

Figure 1a demonstrates time-integrated PL spectra corresponding to the GaN/AlN single QWs of different nominal thickness varied in the range 1-2 ML. The colored spectra are taken in the same areas where the TEM images have been obtained. The PL spectra revealed non-monotonous dependence of the emission intensity on GaN thickness, with maximum wavelength at 235-240 $\mathrm{nm}$. Such a behavior is presumably related to a competition between two trends: an increase of electronhole wave-function overlap and an increase of a relative part of TM polarized emission. The first process results from suppression of the quantum-confined Stark effect, while reducing GaN thickness, and leads to the increase of both radiative recombination rate and emission intensity. The second process results from the increase of the quantized hole level energy, accompanied by a progressively increased contribution of the hole states from the crystal-field split valence band; this leads to hampering of light extraction from the front surface of the hetero-structure.

Figure $1 \mathrm{~b}$ shows the PL decay curves measured in $2 \mathrm{ML}, 1.5 \mathrm{ML}$, and $1 \mathrm{ML}$ thick QWs (curves are normalized by maximum and arrows in Fig. 1a indicate the corresponding detection wavelengths). Temporal behavior of the emission can be described by fast and slow decaying components with time constants $\sim 0.2-1 \mathrm{~ns}$ and $\sim 7-20 \mathrm{~ns}$, respectively. The first one dominates in the thicker GaN insertions and becomes faster and less intense when $\mathrm{GaN}$ thickness decreases down to a single monolayer. The second one, on the contrary, becomes dominating in the thinnest GaN insertions. While the faster PL component can be associated with radiative recombination of localized exitons in the QW, the emergence of the slower PL component assumes a decrease of the electron-hole overlap due to presumable formation of type II band line ups.

Figure 2 shows a typical cross-sectional TEM image of the GaN/AIN hetero-structure (a) and HAADF STEM images of 1- 5 ML thick GaN QWs (b-d) embedded in AlN matrix. The bright-field 
TEM image (Fig. 2a) demonstrates the reduction of threading dislocations density due to ultra-thin GaN layers inserted in AlN layers [3]. This leads to dislocation bending into interfaces with their further annihilation ("blocking" effect). The HAADF STEM images of GaN QWs (Figs. 2, b-c) confirm that the thickness of QWs can be maintain with a high accuracy. However, one can see some variations in the $\mathrm{Al} / \mathrm{Ga}$ composition within each $\mathrm{QW}$ that may affect the optical properties. Thus a combination of PL studies with STEM analysis is a valuable tool in understanding structure - optical properties relationship that will lead to development of high efficiency deep-UV photonic devices [4].

\section{References:}

[1] S.M. Islam et al, Appl. Phys. Lett. 110 (2017), 041108.

[2] X. Rong et al, Adv. Mater. 28 (2016), p. 7978.

[3] S. V. Ivanov et al, Semicond.Sci.Technol. 29 (2014), 084008.

[4] The authors would like to acknowledge the supported by the Government of the Russian Federation (Project \# 14.W03.31.0011) and use of NDIIF electron microscopy facilities at University of Notre Dame.

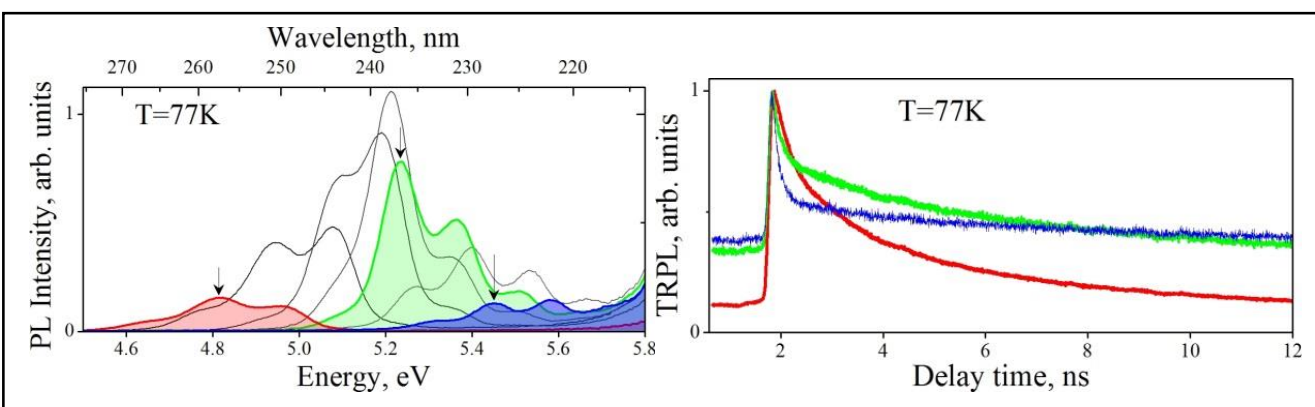

Figure 1. Time integrated PL spectra (a) and PL decay curves (b)

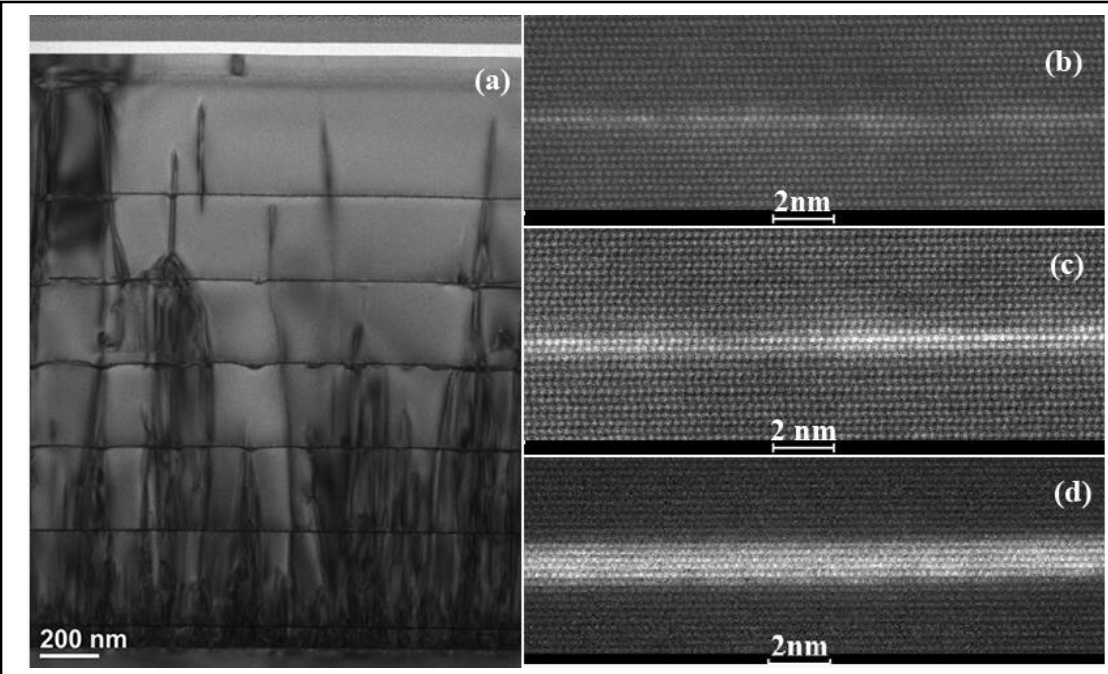

Figure 2. BF cross-sectional TEM image of the GaN/AIN hetero-structure (a) and HAADF STEM images of 1-2 ML (b), 2-3 (c) and 4-5 ML (d) thick GaN QWs in AlN matrix 